\title{
Suppressive actions of volatile anaesthetics on the response capability in cats
}

Koichi Tsushima MD, Koh Shingu $M D$, Sakahiro Ikeda MD, Hiromi Kimura MD, Koh Yamada MD, Kohei Murao MD

Purpose: Suppression of response to a given stimulus by anaesthetics might be considered as a summation of the suppression of basal (pre-stimulus) activity and response capability (increased by stimulus). Anaesthetic suppression of each component in brain and cardiovascular variables by halothane, isoflurane or sevoflurane was compared in cats.

Methods: Thirty cats were allocated to one of three groups $(n=10$ in each) according to the anaesthetic given. The sciatic nerve was stimulated after maintaining the end-tidal concentration of the anaesthetic at 1.3 or $2.0 \mathrm{MAC}$ for at least $30 \mathrm{~min}$. Cortical electroencephalogram (EEG), multi-unit activity in the mid-brain reticular formation (R-MUA), mean arterial pressure (MAP) and heart rate (HR) were measured before and after electrical sciatic nerve stimulation.

Results: The EEG patterns and R-MUA indicated greater suppression of activity in the brain by isoflurane $(3 \mid \pm 4 \%$ of awake state at $1.3 \mathrm{MAC}$, mean \pm SEM) and sevoflurane $(38 \pm 5 \%)$ than by halothane $(61 \pm 5 \%, P<0.05)$, before stimulation. The R-MUA following the stimulation was not different among agents. The MAP and HR were not different among groups before stimulation, but following stimulation were greater in the sevoflurane group ( $137 \pm 9$ and $103 \pm 9 \mathrm{mmHg}$ at 1.3 and $2.0 \mathrm{MAC}$ ) than in the halothane group (103 \pm 5 and $76 \pm 3 \mathrm{mmHg}, P<0.05)$.

Conclusion: Isoflurane and sevoflurane have greater suppressive action on the basal CNS activity than halothane at the same MAC, and that these two anaesthetics have a weak suppressive action on the response capability to peripheral stimulation.

Objectif : La suppression de la réponse à un stimulus par les anesthésiques peut être considérée comme la sommation de la suppression de l'activité basale (pré-stimulus) et de la capacité de réponse (augmentée par le stimulus). La suppression par l'anesthésie de chacune de ces composantes au niveau du cerveau ainsi que des variables cardiovasculaires a été comparée chez le chat pour l'halothane, l'isoflurane et le sevoflurane.

Méthodes : Trente chats ont été répartis en 3 groupes $(n=10)$ selon l'agent anesthésique administré. Après avoir maintenu la concentration en fin d'expiration de l'agent anesthésique à I,3 et 2,0 CAM pour 30 minutes, on a stimulé le nerf sciatique. Avant et après cette stimulation, on a enregistré l'électroencéphalogramme cortical (EEG), l'activité plurisynaptique de la formation réticulée mésencéphalique (R-MVA), la pression artérielle moyenne (PAM) et la fréquence cardiaque $(F C)$.

Résultats : Les tracés d'EEG et de R-MVA indiquent une plus grande dépression de l'activité cérébrale avant stimulation par l'isollurane ( $31 \pm 4 \%$ des valeurs d'éveils à 1,3 CAM, moyenne \pm écart type moyen) et le sevoflurane (38 $\pm 5 \%$ ) que par l'halothane $(61 \pm 5 \%, P<0,05)$. À la suite de stimulation, la R-MVA ne montrait pas de différence entre les agents. Avant stimulation, la PAM et la FC des différents groupes n'affichaient aucune différence, mais après stimulation les valeurs de PAM étaient plus élevées dans le groupe sevoflurane (137 \pm 9 et $103 \pm 9 \mathrm{mmHg}$ à 1,3 et 2,0 CAM) que dans le groupe halothane $(103 \pm 5$ et $76 \pm 3 \mathrm{mmHg}, P<0,05)$.

Conclusion : Pour une même CAM, l'isoflurane et le sevoflurane ont une action suppressive plus intense sur l'activité de base du SNC que l'halothane; d'autre part, ces deux agents ont une action suppressive faible de la réponse à une stimulation périphérique.

From the Department of Anesthesiology, Kansai Medical University, Fumizono-cho 10-15, Moriguchi, Osaka 570, Japan.

Address for correspondence: Koh Shingu $\mathrm{MD}$, Department of Anesthesiology, Kansai Medical University, Fumizono-cho 10-15, Moriguchi, Osaka 570, Japan; Phone: 81-6-992-1001; Fax: 81-6-998-9625.

This study was supported in part by Grants-in-Aid for Scientific Research No. 07771275 and 08457415 , from the Ministry of Education, Science and Culture, Japan.

Accepted for publication December 5, 1997. 


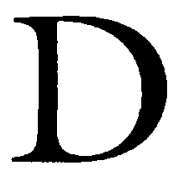

URING isoflurane or sevoflurane anaesthesia, arterial blood pressure may increase unexpectedly after tracheal intubation or skin incision, while the blood pressure in the pre-stimulus period appears to be sufficiently suppressed to suggest an adequate plane of anaesthesia. ${ }^{1 a}$ By contrast, changes of blood pressure from the pre-stimulus value can be easily predicted during halothane anaesthesia. This clinical impression of a difference between halothane and isoflurane or sevoflurane led us to compare the supression of basal (pre-stimulus) activity from response capability with the three agents. Anaesthetic effects on the cardiovascular system are mediated, in part, through their direct actions on the heart and vasculature and their effects on the endocrinological system. However, a major factor affecting cardiovascular effects of anaesthetics may be their actions on the central nervous system (CNS), because the cardiovascular system is under both tonic and phasic control of the nervous system. The degree of neuronal firing in the mid-brain reticular formation, which reflects a non-specific acitivity of the brain, can be evaluated in a quantitative manner using a multi-unit activity (MUA) recording method. ${ }^{2}$ We hypothesised that the potency of suppressive actions of an anaesthetic agent on the basal activity and response capability was different for each agent, and the cardiovascular variables correlated with CNS activity.

The purpose of this study was to compare the suppressive actions of three volatile anaesthetics, halothane, isoflurane and sevoflurane, at the same MAC, on basal activity and response capability of the circulatory system and CNS. Arterial blood pressure (ABP), HR, cortical electroencephalogram (EEG), and MUA in the midbrain reticular formation (R-MUA) were recorded before, and after, electrical stimulation of the sciatic nerve in cats.

\section{Methods}

Thirty cats, $3.0-3.5 \mathrm{~kg}$, were used in this study, which was approved by the Institutional Committee on Animal Research. They were anaesthetised with $40 \mathrm{mg} \cdot \mathrm{kg}^{-1}$ pentobarbital ip for placement of electrodes at least two weeks before the start of the study. Stainless steel screws, $2.0 \mathrm{~mm}$ in diameter, were inserted in the frontal bone of the skull (reference electrode) and over the temporal cortex to record the cortical EEG. Parallel stainless steel wire electrodes, $0.2 \mathrm{~mm}$ in diameter, insulated with epoxylite resin except at the tips, with a ver-

\footnotetext{
a Yamada $K$, Shingu $K$, Kimura $H$, et al. Circulatory and catecholamine responses to tracheal intubation and skin incision during sevoflurane, isoflurane, or halothane anesthesia. J Anesth 1997; 11: 111-6.
}

tical separation of $0.5-1.0 \mathrm{~mm}$ at the tips, were inserted bilaterally in the midbrain reticular formation (A2; $\mathrm{L} 3 ; \mathrm{H} ;-2$, according to the atlas of Snider and $\mathrm{Niemer}^{3}$ ) to record the R-MUA. The same wire electrodes were inserted into the dorsal hippocampus (A2; L8; H9) and the medial amygdala (A12; L9; H6). The electrodes were connected to a socket which was fixed to the skull with dental cement.

The EEG was recorded on an eight-channel polygraph (Nihon-Koden polygraph, AB621G, Japan) and the R-MUA and ABP on an oscillograph (NihonKoden, WT685G, Japan). The firing of a population of reticular neurons was measured using an MUA technique described previously. ${ }^{2}$ With this technique, neuronal discharges were picked up from an area of approximately $1 \mathrm{~mm}$ radius around the tip of the electrode. ${ }^{4}$ Changes in the MUA level with the administration of the anaesthetic agents or the stimulation were expressed as a percent of the activity in the control (awake) period.

The animals were allocated to one of three groups of 10 and each group was given a different volatile anaesthetic; halothane, isoflurane or sevoflurane. Following control recording of the EEG and R-MUA in an awake state, the cats were anaestherised with the appropriate agent in oxygen in a $50 \mathrm{~L}$ anaesthesia box. Tracheal intubation was facilitated by $1 \mathrm{mg} \cdot \mathrm{kg}^{-1}$ vecuronium iv, supplemented as required during the study. The lungs were ventilated mechanically using a non-rebreathing method (Acoma Animal Respirator, AR-300, Japan). End-tidal concentrations of carbon dioxide and anaesthetic agents were measured using an infra-red anaesthetic monitor (Capnomac Ultima, Datex, Finland), which was calibrated every experimental day. End-tidal carbon dioxide tension was maintained within the range $30-35 \mathrm{mmHg}$ by adjusting the tidal volume. A cannula was inserted into the femoral artery to permit monitoring of ABP. Another catheter was placed in a forepaw vein for administration of fluids and drugs. A rectal thermistor (Termo-Finer, TN-DN, Japan) was inserted and the rectal temperature was maintained at $37-39^{\circ} \mathrm{C}$ with a warm water mattress and heating lamp. For sciatic nerve stimulation, the sciatic nerve was exposed, its peripheral side was cut and the nerve was then placed on two platinum electrodes; $5 \mathrm{~mm}$ apart. The sciatic nerve was immersed in paraffin warmed at $37^{\circ} \mathrm{C}$.

The sciatic nerve was stimulated electrically using a stimulator (Nihon-Koden, SEN-3301, Japan). The supramaximal intensity of the stimuli (approximately $30 \mathrm{~V}$ ) was determined by measuring the increase of MUA at 1.3 MAC of each agent, and was used during the experiment for each cat. The stimuli were $20 \mathrm{~Hz}$ in frequency for $15 \mathrm{sec}, 0.5 \mathrm{msec}$ in duration, and were 
square waves in shape. The end-tidal concentration of the anaesthetic agent was maintained at 1.3 MAC for at least $30 \mathrm{~min}$ and the sciatic nerve was then stimulated. The end-tidal concentration of anaesthetic was then increased to 2.0 MAC and maintained for $30 \mathrm{~min}$, and the sciatic nerve was stimulated again. The MACs were $1.2 \%$ for halothane, $1.6 \%$ for isoflurane ${ }^{5}$ and $2.6 \%$ for sevoflurane. ${ }^{b}$ The EEG, R-MUA, and ABP were recorded continuously. Mean arterial pressure (MAP) and $H R$ were measured before stimulation, immediately at the end of stimulation, and at one, two, three, four and five minutes after stimulation.

Data are presented as mean values \pm SEM. The data in each group were analysed using repeated measures analysis of variance and the Bonferroni/Dunn test. The data among groups were analysed with ANOVA and the Bonferroni/Dunn test. Differences at $P<0.05$ were considered as significant.

\section{Results}

Representative recordings of the EEG in the cortex, amygdala and hippocampus, R-MUA, and ABP in cats given 1.3 MAC of halothane and 2.0 MAC of sevoflurane are shown in Figures 1 and 2, respectively.

\section{EEG in the cortex}

The cortical EEG in any group before stimulation could be classified into one of four patterns; high amplitude slow waves, burst suppression, isoelectric waves, or sporadic spikes. The number of cats in each group which showed a given cortical EEG pattern before stimulation is shown in the Table below. In cats given halothane at 1.3 MAC, pre-stimulation EEG showed that the dominant waves were high amplitude slow waves $(100-200 \mu \mathrm{V}$ and $2-4 \mathrm{~Hz})$, which were superimposed with low amplitude faster waves $(30-50$ $\mu \mathrm{V}$ and $16-20 \mathrm{~Hz}$ ). Sciatic nerve stimulation changed the dominant waves of the EEG to low amplitude

TABLE Cortical EEG patterns before stimulation

\begin{tabular}{lllllll}
\hline & Halothane & \multicolumn{2}{l}{ Isoflurane } & \multicolumn{2}{l}{ Sevoflurane } \\
\hline MAC & 1.3 & 2.0 & 1.3 & 2.0 & 1.3 & 2.0 \\
High-amplitude slow waves & 10 & 10 & 3 & 0 & 4 & 0 \\
Burst suppression & 0 & 0 & 4 & 0 & 2 & 0 \\
Isoelectric & 0 & 0 & 3 & 5 & 3 & 3 \\
Sporadic spikes & 0 & 0 & 0 & 5 & 1 & 7 \\
\hline
\end{tabular}

Values are numbers of cats showing a given cortical EEG pattern ( $\mathrm{n}=10$ in each agent)

b Doi $M$, Yunoki $H$, Ikeda $K$. The minimum alveolar concentration of sevoflurane in cats. J Anesth 1988; 2: 113-4. faster waves $(40-60 \mu \mathrm{V}$ and $15-20 \mathrm{~Hz})$. An increase in concentration of halothane to $2.0 \mathrm{MAC}$ attenuated the faster waves and made the high amplitude slow waves more obvious. Sciatic nerve stimulation reduced the amplitude to $50-100 \mu \mathrm{V}$ and increased the frequency to $15-20 \mathrm{~Hz}$ in seven cats given halothane at 2.0 MAC, whereas stimulation did not induce any obvious changes in the EEG of the remaining three cats given halothane.

HALOTHANE 1.3 MAC

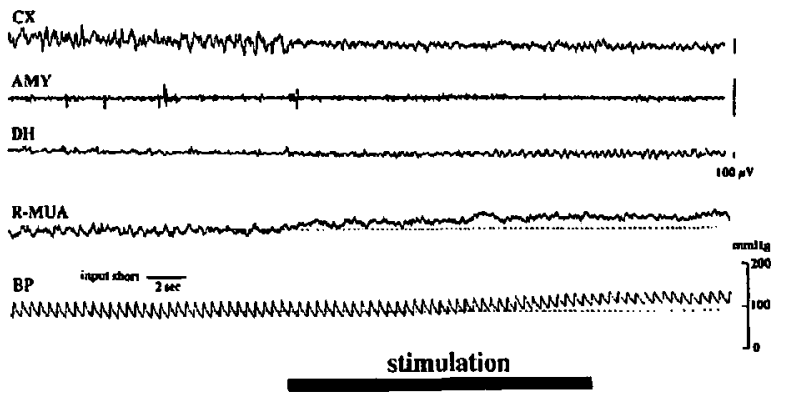

FIGURE I The EEG in the cortex, amygdala, and hippocampus, R-MUA, and arterial blood pressure during electrical stimulation of the sciatic nerve with 1.3 MAC halothane in a cat. CX: cortex, AMY: amygdala, DH: dorsal hippocampus, R-MUA: multi-unit activity in the midbrain reticular formation, BP: arterial blood pressure. The cortical EEG before stimulation shows high amplitude and slow waves. Sciatic nerve stimulation decreases the amplitude and increases the frequency of the cortical EEG, and increases RMUA and arterial blood pressure. EEG in the dorsal hippocampus showed rhythmic waves following stimulation, indicating activation. The dotted lines indicate the levels of basal R-MUA and MAP.

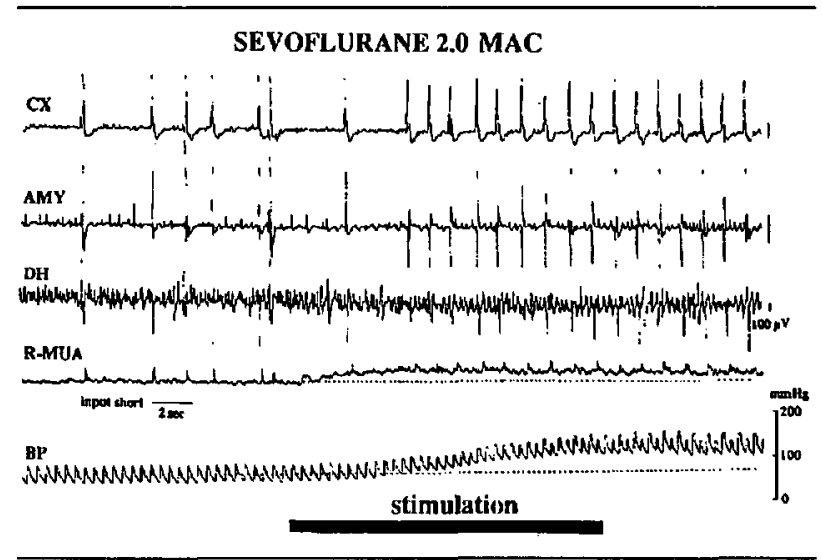

FIGURE 2 The EEG in the cortex, anygdala, and hippocampus, R-MUA, and arterial blood pressure during electrical stimulation of the sciatic nerve with 2.0 MAC sevoflurane in a cat. CX: cortex, AMY: amygdala, DH: dorsal hippocampus, R-MUA: multi-unit activity in the midbrain reticular formation, BP: arterial blood pressure. The cortical EEG shows sporadic spikes before stimulation. Electrical stimulation of the sciatic nerve increases the occurrence of spikes, R-MUA, and arterial blood pressure. The dotted lines indicate the levels of basal R-MUA and MAP. 
At 1.3 MAC isoflurane, sciatic nerve stimulation reduced the amplitude and increased the frequency of the waves in cats which showed high amplitude slow waves. In cats which showed burst suppression or isoelectric EEG, the stimulation either reduced the duration of the isoelectric waves or abolished them completely. During administration of isoflurane at 2.0 MAC, sciatic nerve stimulation changed the isoelectric waves to sporadic spikes in two cats, a high voltage slow wave pattern in one, and no change in two cats. Sporadic spikes seen pre-stimulus were still present after stimulation and did not appear to have changes in frequency.

During administration of sevoflurane at 1.3 MAC, stimulation abolished the period of isoelectric waves and all cats showed high amplitude slow waves. During administration of sevoflurane at 2.0 MAC, the stimulation changed the isoelectric waves to a slow wave pattern in one cat whereas no change was observed in two. In cats which showed sporadic spikes, the frequency of the spikes was increased by the stimulation.

\section{Mid-brain reticular firing}

Anaesthetics suppressed the basal R-MUA. The suppression of the R-MUA by halothane was less than with isoflurane and sevoflurane at both 1.3 and 2.0 MAC $(P<0.05)$. Sciatic nerve stimulation increased the R-MUA in all groups $(P<0.05)$ (Figure 3$)$. The

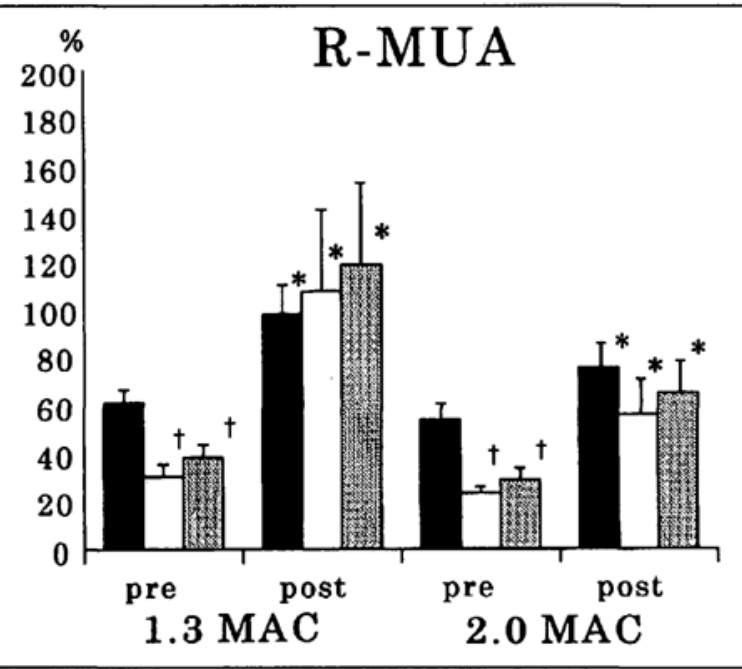

FIGURE 3 Neuronal firing in the mid-brain reticular formation measured with multi-unit activity (R-MUA) before and after stimulation.

Values are mean \pm SEM, $\mathrm{n}=10 .{ }^{*} P<0.05$ vs pre-stimulation, $\dagger P<0.05$ vs halothane; pre: value before stimulation, post: value at the end of stimulation; solid column: halothane, clear column: isoflurane, and hatched column: sevoflurane.

Values of R-MUA are expressed as percentage of activity in the control (awake) state.
R-MUA levels at the end of stimulation were not different among groups.

\section{Mean arterial pressure}

The MAP before sciatic nerve stimulation was not different among cats given any of the anaesthetics at either 1.3 or $2.0 \mathrm{MAC}$. However, the MAP at $2.0 \mathrm{MAC}$ was lower than that during at 1.3 MAC for each of the anaesthetic agents $(P<0.05)$. Sciatic nerve stimulation increased the MAP in all groups except in cats given halothane at $2.0 \mathrm{MAC}(P<0.05)$, and the MAP reached a peak at the end of stimulation and then decreased gradually. The peak MAP at the end of stimulation was higher in the sevoflurane group than in the halothane group during anaesthesia with the agents at both 1.3 and $2.0 \mathrm{MAC}(P<0.05)$ (Figure 4$)$.

\section{Heart rate}

In the isoflurane and sevoflurane groups at $2.0 \mathrm{MAC}$, stimulation increased the HR $(P<0.05)$ with peak values being reached at the end of stimulation. The peak $H R$ at the end of stimulation with isoflurane at 2.0 MAC was higher than with halothane at 2.0 MAC $(P<0.05)$ (Figure 5).

\section{Discussion}

The results of this study showed that isoflurane and sevoflurane caused greater suppression of the basal CNS activity than did halothane. However, suppression of

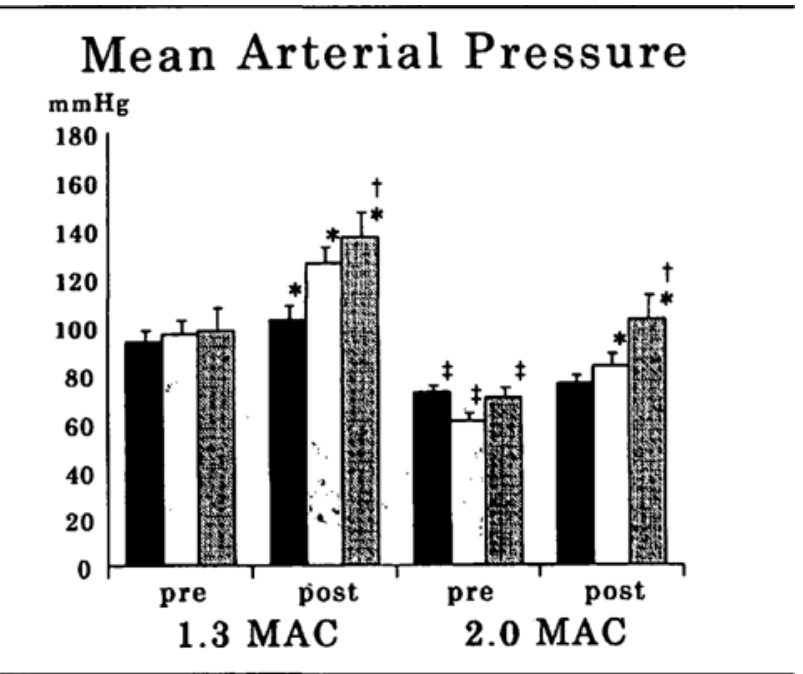

FIGURE 4 Mean arterial blood pressure before and after stimulation.

Values are mean $\pm S E M, \mathrm{n}=10 .{ }^{*} P<0.05 v s$ pre-stimulation $t P<0.05$ vs halothane $¥ P<0.05$ vs $1.3 \mathrm{MAC}$; pre: value before stimulation, post: value at the end of stimulation; solid column: halothane, clear column: isoflurane, and hatched column: sevoflurane. 
the response capability should be greater in halothane than with isoflurane and sevoflurane anaesthesia, because CNS activity following sciatic nerve stimulation did not differ among the agents. The MAP following stimulation also increased markedly during sevoflurane anaesthesia, while there was no difference in MAP with any of the agents before stimulation.

The basal cortical EEG at the pre-stimulated state was different among anaesthetic agents at the same MAC. The EEG patterns with halothane were similar to those seen with lower concentrations of isoflurane and sevoflurane. These similarities indicate that suppression of the brain, reflected on the cortical EEG, during isoflurane and sevoflurane anaesthesia is greater than that during halothane anaesthesia at the same MAC. The R-MUA showed greater suppression of the CNS during isoflurane and sevoflurane anaesthesia than during halothane anaesthesia. Ogawa et al. also showed greater suppression of the R-MUA during isoflurane and enflurane anaesthesia than during halothane anaesthesia. ${ }^{6}$ These findings in cortical EEG and R-MUA suggest that basal CNS activity is suppressed to a greater degree during isoflurane and sevoflurane anaesthesia than by halothane.

Although suppression in the CNS activity was different among anaesthetics, MAP and HR were not different at the pre-stimulated state. The discrepancy in suppression of the activity of the CNS, and MAP or $H R$, may be caused by different potencies of the anaesthetics on the direct action of the cardiovascular system.

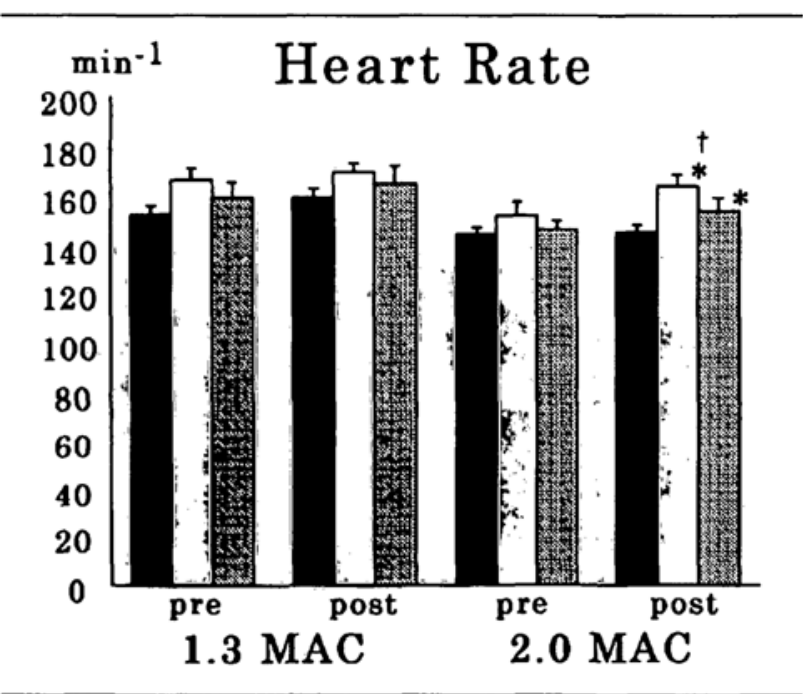

FIGURE 5 Heart rate before and after stimulation.

Values are mean $\pm S E M, n=10$.

${ }^{*} P<0.05$ vs pre-stimulation, ${ }^{\dagger} P<0.05$ vs halothane; pre: value before stimulation, post: value at the end of stimulation. solid column: halothane, clear column: isoflurane, and hatched column: sevoflurane.
Suppression of basal R-MUA by halothane was less than that observed with isoflurane and sevoflurane, but the R-MUA levels following sciatic nerve stimulation were not different among the three groups. This indicates that the suppressive action of halothane on the response capability of the CNS is more potent than that seen with isoflurane or sevoflurane, and correlates well with our previous findings. ${ }^{6-8}$ Ogawa et al. reported that halothane had a greater suppressive action on the visual evoked potentials in cats when compared with isoflurane and enflurane. ${ }^{6}$ Sevoflurane had a similar action on the somatosensory evoked potential to enflurane in cats. ${ }^{7}$ Shingu et al. showed that halothane suppressed the bradykinin-induced neuronal response in the lumbar lateral funiculus in cats in which cervical spinal cord was transected, whereas enflurane had no effect. $^{8}$

Although a discrepancy existed between the suppression of CNS activity and MAP pre-stimulation, the response in ABP to sciatic nerve stimulation correlated with the response observed in R-MUA. The present study indicated that halothane is more potent than isoflurane and sevoflurane in suppressing the $\mathrm{ABP}$ response when these anaesthetics are compared on a MAC basis. Roizen et al. defined the potency of an anaesthetic in blocking the adrenergic response to skin incision as MAC-BAR, and demonstrated no difference between anaesthetics in the ratio of MAC-BAR to MAC. The value of MAC-BAR was 1.45 MAC for halothane and 1.60 MAC for enflurane. ${ }^{9}$ The patients in their study, however, received $60 \%$ nitrous oxide with the anaesthetics tested. Nitrous oxide has a potent analgesic action and might mask any differences between the potencies of the anaesthetics in suppressing adrenergic and cardiovascular responses to noxious stimuli. The present study was performed without analgesics or nitrous oxide and revealed that the ratios of MAC-BAR to MAC might vary with different agents. Zbinden et al. also reported that isoflurane has weak actions to suppress hemodynamic reactions to strong stimulation in humans. ${ }^{1}$

Suppression of a response may depend on the type of response observed. We chose circulatory variables and CNS activity. The suppression of body movement by anaesthetics in response to noxious stimuli may involve suppression of the sensory systems, the central integrating systems and the motor nervous system as well as the effector, skeletal muscles. If a different response is evaluated, the systems involved in the evoked responses are different. Volatile anaesthetics do not have identical actions on the various networks in the nervous system and different anaesthetics may have diverse actions on a given system. Thus, the suppressive actions of anaes- 
thetic agents may vary if different responses are observed. However, the present results indicate that general suppressive actions of anaesthetics on the response capability in the CNS contribute a great part in determining the MAP changes induced by stimuli.

The current study revealed weak suppressive actions of isoflurane and sevoflurane on response capability. In order to suppress response induced by stimuli, higher concentrations of agents are required, which cause greater basal suppression. We recommend that these agents should be used with nitrous oxide, opioids and/or other analgesic regimen to suppress reactions to noxious stimulation without depression at the basal (pre-stimulated) period.

We conclude that isoflurane and sevoflurane have greater suppressive actions on the basal CNS activity than halothane at the same MAC, and that these two anaesthetics have a weak suppressive action on the response capability to peripheral stimulation.

\section{References}

1 Zbinden AM, Petersen-Felix S, Thomson DA. Anesthetic depth defined using multiple noxious stimuli during isoflurane/oxygen anesthesia. II. Hemodynamic responses. Anesthesiology 1994; 80: 261-7.

2 Mori K, Kawamata M, Mitani H, ramazaki r, Fujita $M$. A neurophysiologic study of ketamine anesthesia in the cat. Anesthesiology 1971; 35: 373-83.

3 Snider RS, Niemer WT. A Stereotaxic Atlas of the Cat Brain. Chicago: University of Chicago Press, 1961.

4 Halas ES, Beardsley JV. Specificity of multiple unit activity in the sensory nuclei of cats. Physiol Behav 1968; 3: 275-9.

5 Drummond JC, Todd MM, Shapiro HM. Minimum alveolar concentrations for halothane, enflurane, and isoflurane in the cat. J Am Vet Med Assoc 1983; 182: 1099-101.

6 Ogawa T, Shingu K, Shibata M, Osawa M, Mori K. The divergent actions of volatile anaesthetics on background neuronal activity and reactive capability in the central nervous system in cats. Can J Anaesth 1992; 39: 862-72.

7 Osawa $M$, Shingu $K$, Murakawa $M$, et al. Effects of sevoflurane on central nervous system electrical activity in cats. Anesth Analg 1994; 79: 52-7.

8 Shingu $K$, Osawa $M$, Omatsu $\Upsilon$, Komatsu $T$, Urabe $N$, Mori $K$. Naloxone does not antagonize the anestheticinduced depression of nociceptor-driven spinal cord response in spinal cats. Acta Anaesthesiol Scand 1981; 25: 526-32.

9 Roizen MF, Horrigan RW, Frazer BM. Anesthetic doses blocking adrenergic (stress) and cardiovascular responses to incision - MAC BAR. Anesthesiology 1981; 54: 390-8. 